\title{
Characteristics and genetic testing outcomes of patients with clinically suspected paraganglioma/ pheochromocytoma (PGL/PCC) syndrome in Singapore
}

\author{
Kay Reen Ting ${ }^{1}$, Pei Yi Ong ${ }^{1}$, Samuel Ow Guan Wei, ${ }^{1,2}$, Rajeev Parameswaran ${ }^{3}$, Chin Meng Khoo ${ }^{2,4}$,
} Doddabele Srinivasa Deepak ${ }^{4}$ and Soo-Chin Lee ${ }^{1,5^{*}}$ (D)

\begin{abstract}
Background: Hereditary paraganglioma (PGL) and pheochromocytoma (PCC) syndromes are rare conditions, with limited data on spectrum of causative gene variants of these syndromes in Asian patients.

Methods: We describe the clinical characteristics and genetic testing outcomes of patients with suspected hereditary PGL/PCC who were referred to a tertiary cancer genetics clinic in Singapore.

Results: Among 2196 patients with suspected hereditary cancer syndrome evaluated the cancer genetics clinic from 2000 to 2019, 13/2196 (0.6\%) patients fulfilled clinical suspicion for hereditary PGL/PCC syndrome. After genetic counselling, 10 patients underwent multi-gene next generation sequencing and deletion/duplication analysis, including SDHAF2, SDHA, SDHB, SDHC, SDHD, VHL, NF1, RET, MAX, and TMEM127. Seven of 10 patients (70\%) were identified to carry pathogenic variants, including 3 unrelated Chinese patients with head and neck PGL who carried the same SDHD: c.3G > C (p.Met1lle) variant that was previously reported to be a possible founder variant in Chinese, and 3 patients with urogenital PGL and 1 patient with retroperitoneal PGL who carried different SDHB variants. Variant carriers were younger, more likely to present with multiple tumours, or have family history of paraganglioma or pheochromocytoma, than non- variant carriers.
\end{abstract}

Conclusion: Hereditary PGL/PCC accounts for only $0.6 \%$ of patients seen in an adult cancer genetics clinic in Asia. SDHD and SDHB genes remain the most important causative genes of hereditary PGL/PCC in Asia even when patients are tested with multi-gene NGS panel.

Keywords: Hereditary paraganglioma, Pheochromocytoma, Genetic testing, SDHB, SDHD, Chinese

\footnotetext{
* Correspondence: csilsc@nus.edu.sg

'Department of Haematology-Oncology, National University Cancer Institute,

Singapore (NCIS), National University Health System, 1E Kent Ridge Road,

Singapore 119228, Singapore

${ }^{5}$ Cancer Science Institute, Singapore, Singapore

Full list of author information is available at the end of the article
}

(c) The Author(s). 2020 Open Access This article is licensed under a Creative Commons Attribution 4.0 International License, which permits use, sharing, adaptation, distribution and reproduction in any medium or format, as long as you give appropriate credit to the original author(s) and the source, provide a link to the Creative Commons licence, and indicate if changes were made. The images or other third party material in this article are included in the article's Creative Commons licence, unless indicated otherwise in a credit line to the material. If material is not included in the article's Creative Commons licence and your intended use is not permitted by statutory regulation or exceeds the permitted use, you will need to obtain permission directly from the copyright holder. To view a copy of this licence, visit http://creativecommons.org/licenses/by/4.0/. The Creative Commons Public Domain Dedication waiver (http://creativecommons.org/publicdomain/zero/1.0/) applies to the data made available in this article, unless otherwise stated in a credit line to the data. 


\section{Introduction}

Pheochromocytomas (PCC) and paragangliomas (PGL) are rare neuroendocrine tumours with incidence rate occurring around 2 to 8 cases per million person years [1, $2]$, affecting both genders equally and commonly occurring in the third to fifth decades of life. The 2017 World Health Organisation (WHO) classification of Endocrine Tumour and American Joint Committee on Cancer has classified these tumours based on the anatomy and biochemical features [3]. Head and neck paragangliomas (HN-PGL) involve the skull base, neck and upper mediastinum; with the commonest site of tumour occurring above the bifurcation of carotid arteries. This group of paragangliomas are typically parasympathetic and nonsecretory. Extra-adrenal paragangliomas found in the lower mediastinum, abdomen such as the organ of Zuckerkandl and pelvis (thoraco-abdominal PGL) are typically catecholamine secretors resulting in clinical manifestation of sympathetic-like symptoms. Majority of PGL/PCC are benign cases but approximately $10 \%$ of PCC and around $15-35 \%$ of extra-adrenal abdominal paragangliomas are malignant $[4,5]$. Malignant PGL/ PCC may metastasize to bone, liver and lung, with predicted 5-year survival rates ranging from 12 to $80 \%$ [6, 7]; a meta-analysis reports the overall 5-year mortality rates for patients with metastatic PGL/PCC at 37\% [8].

Historically, about $10 \%$ of PGL/PCCs were associated with hereditary syndromes, but recent discoveries have shown that at least $25-40 \%$ of PGL/PCC are linked to hereditary syndromes with several identified causative genes [9-11]. The classic hallmarks for hereditary PGL/ PCC include an early age of onset, extra-adrenal disease, multiple primary tumours and metastatic tumours. Hereditary PGL/PCCs tend to present about 15 years younger than sporadic cases $[12,13]$.

There are at least 10 genetic syndromes with predisposition to PGL/PCC. The most common are the paraganglioma syndromes due to variants in the succinate dehydrogenase subunit $(S D H)$ genes, $S D H D$, $S D H A F 2, S D H C, S D H B$ and $S D H A$, respectively, that are categorised into 5 types (PGL 1-5), with distinct clinical phenotypes [14]. Among these, variants in PGL1 (SDHD) and PGL4 (SDHB) are most commonly encountered in clinical practice. PGL1 $(S D H D)$ is predominantly associated with head and neck PGL that frequently presents as multifocal disease and rarely malignant, while PGL4 (SDHB) presents with head and neck or thoraco-abdominal PGLs with only 20$25 \%$ being multifocal but behave more aggressively with $\sim 30 \%$ being malignant. Adrenal pheochromocytomas and renal cell carcinomas occur in both PGL1 and PGL4, at $\sim 10-25 \%$ and $8-14 \%$ respectively, while gastrointestinal stromal tumours have been reported in PGL1, 3, 4 and 5. In comparison, PGL2 presents almost exclusively with head and neck PGL with rarely other manifestations [14].

Other well-known hereditary syndromes associated with PGL/PCC include multiple endocrine neoplasm type 2 (MEN2), von Hippel-Lindau disease (VHL) and neurofibromatosis type 1 (NF1). In the last decade, newer genes such as $M A X$ and TMEM127 were reported to contribute to hereditary pheochromocytoma and paragangliomas. $M A X$ variants are almost exclusively identified in patients with adrenal pheochromocytoma that are frequently bilateral [15], while TMEM127 variant carriers most commonly present with single adrenal pheochromocytoma, and occasionally multiple head and neck or thoraco-abdominal PGLs.

Studies describing the causative variants and characteristics of hereditary PGL/PCC syndromes in Southeast Asia are limited. We describe a series of patients with suspected hereditary PGL/PCC syndrome who underwent multi-gene panel testing at a Cancer Genetics Program at an academic cancer centre in Singapore.

\section{Materials and methods Study group}

A total of 2196 patients with suspected hereditary cancer syndromes were referred to the National University Cancer Institute, Singapore (NCIS) Adult Cancer Genetic Clinic for genetic counselling and consideration of genetic testing from year 2000 to December 2019. Thirteen of 2196 individuals $(0.6 \%)$ were patients with suspected hereditary paraganglioma or pheochromocytoma (PGL/ PCC) syndrome, presenting either with young onset PGL or PCC at or before age 40, multiple PGL and/or PCCs, and/or family history of PGL/PCC. Patients received genetic counselling and were offered genetic testing using a multi-gene panel test with full-gene sequencing and deletion/duplication analysis using nextgeneration sequencing (NGS) technology, including SDHAF2, SDHA (sequencing changes only), SDHB, SDHC, SDHD, VHL, NF1, RET, MAX, and TMEM127, as well as causative genes of common hereditary breast and colorectal cancer syndromes, including $B R C A 1 / 2$, TP53, and the mismatch repair genes (MLH1, MSH2, MSH6 and PMS2). Cascade testing was offered to firstdegree relatives in patients tested positive for pathogenic germline variants.

\section{Results}

Table 1 summarizes the clinical characteristics of the 13 patients with suspected hereditary PGL/PCC syndrome and Figs. 1 and 2 shows the 7 family pedigree for those patients found with pathogenicvariants. Majority are female $(n=11,84.6 \%)$ and Chinese $(n=9,69.2 \%)$. Six patients (46.2\%) presented with head and neck paraganglioma, five (38.5\%) had extra-adrenal thoraco- 


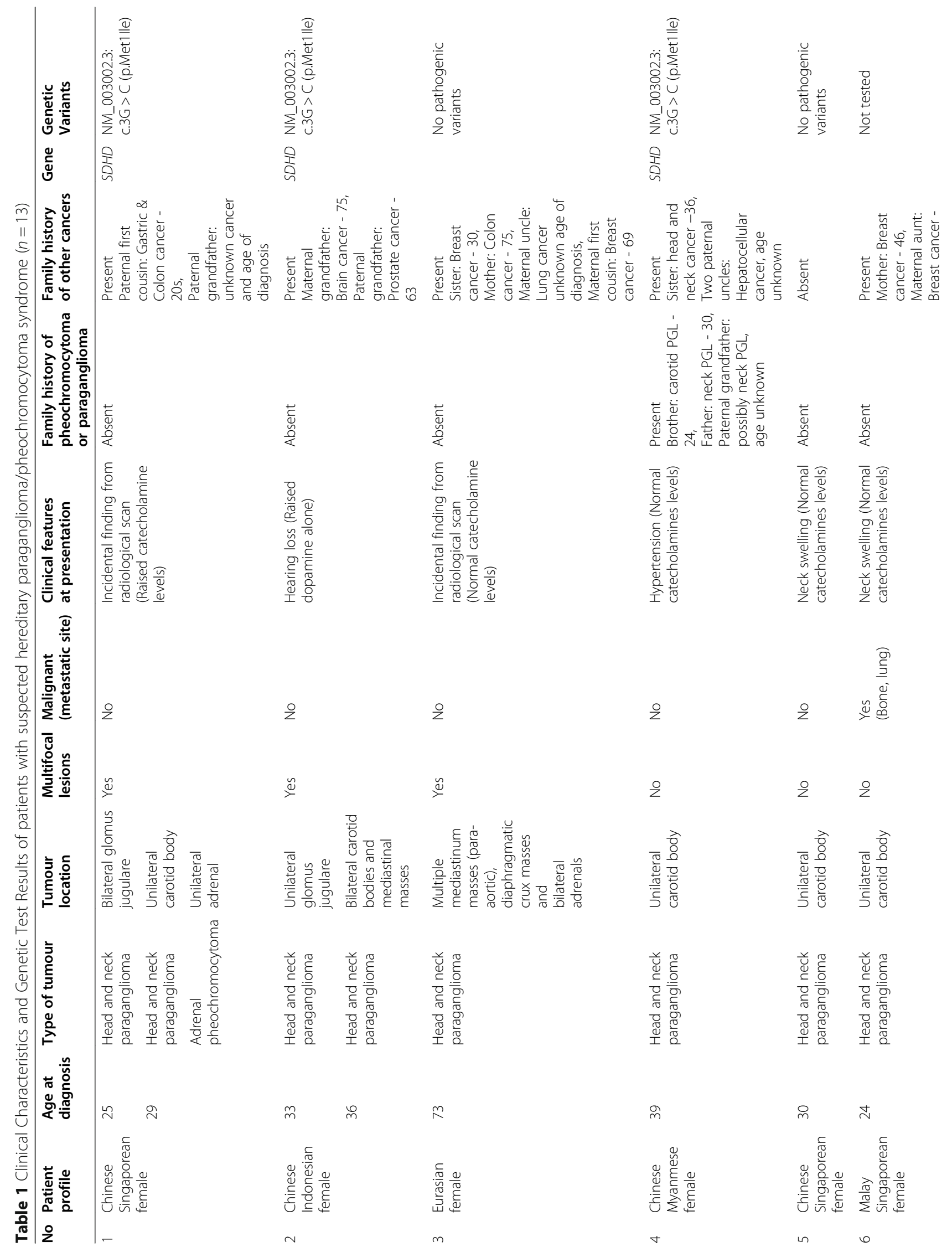




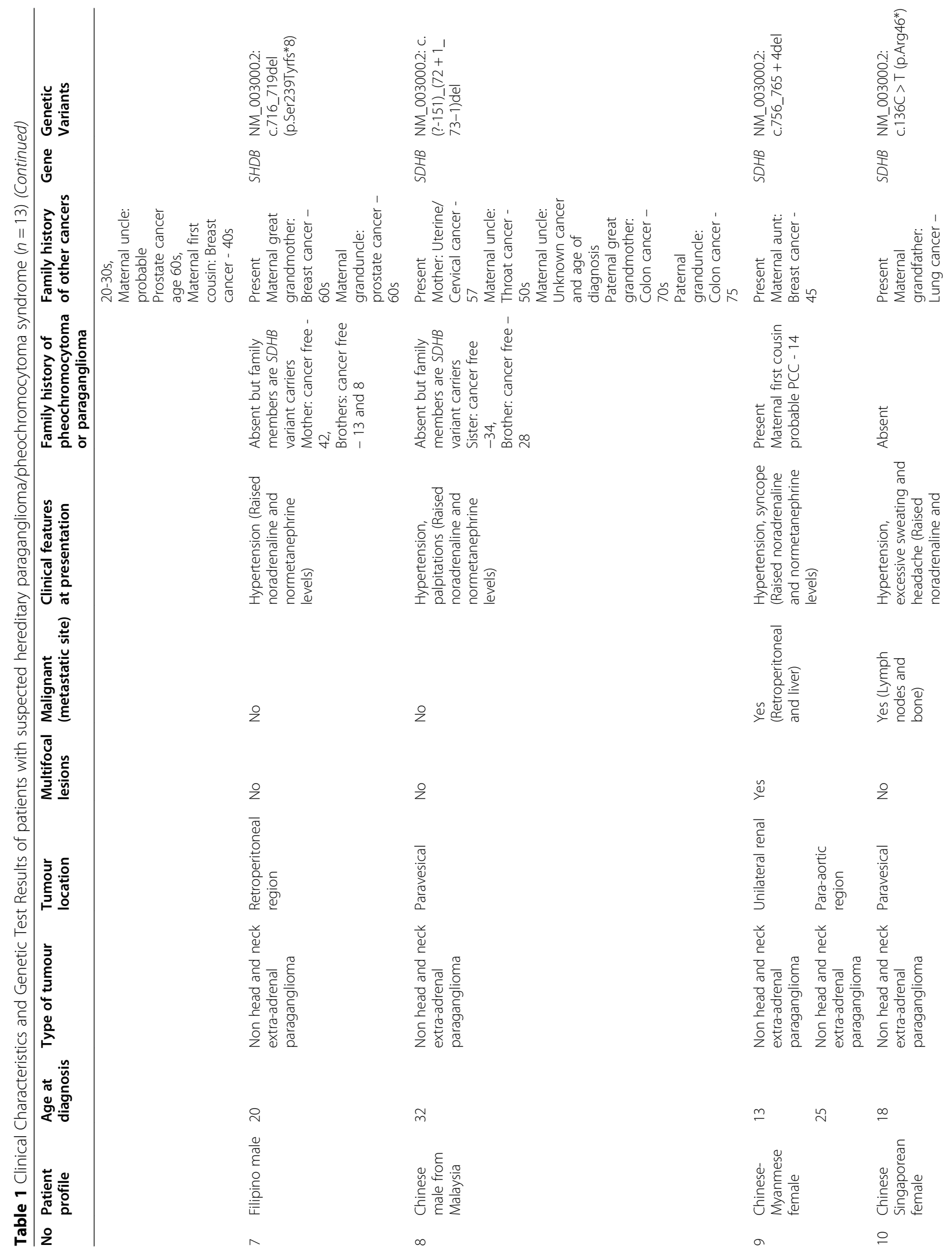




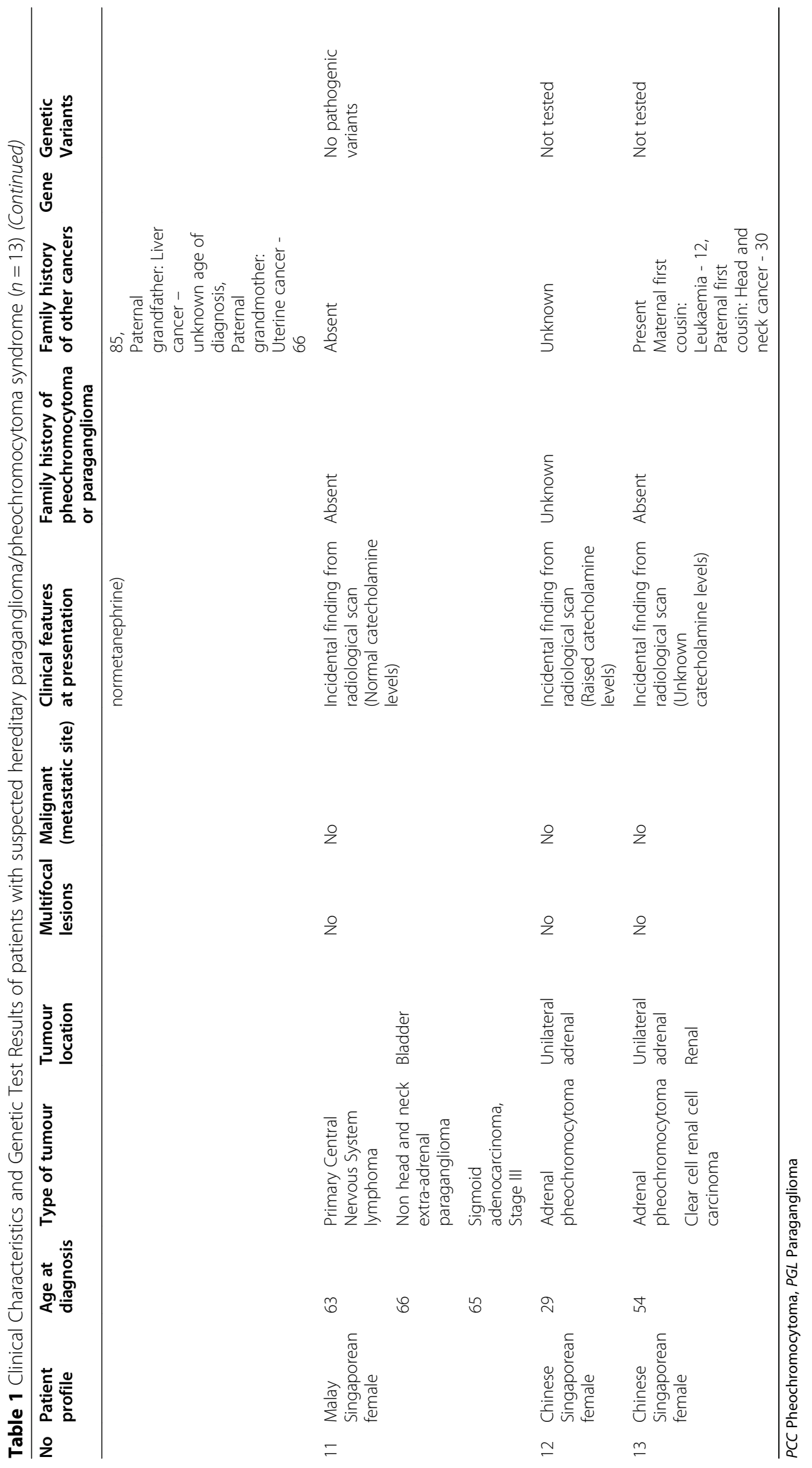


SDHD mutations

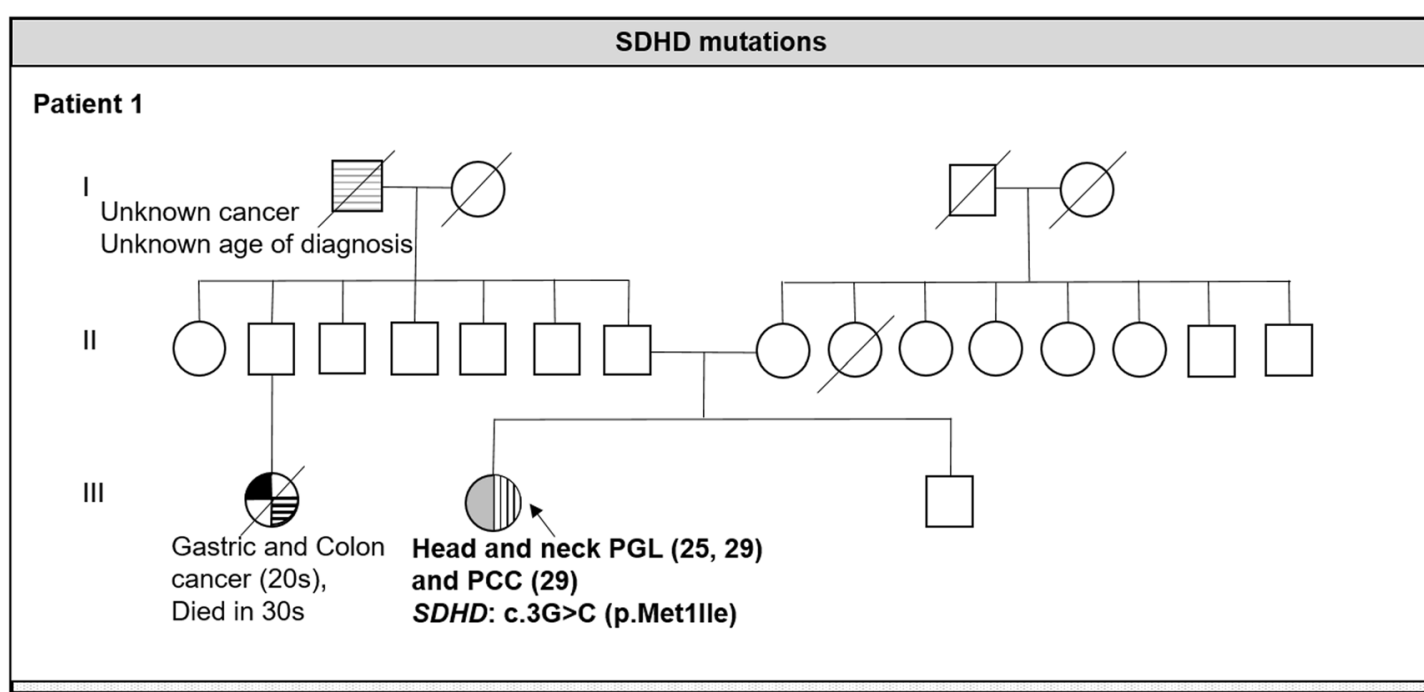

Patient 2

I

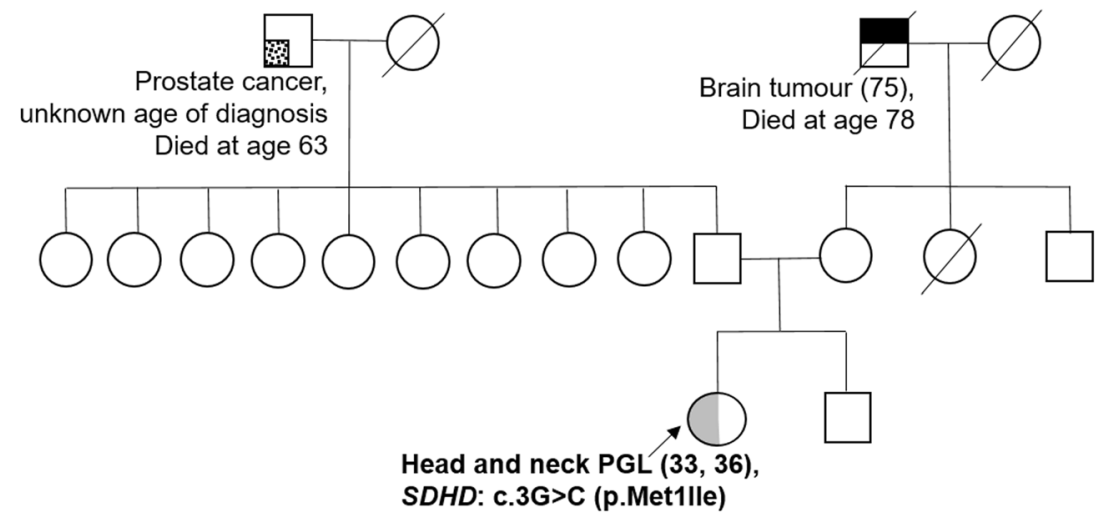

\section{Patient 4}

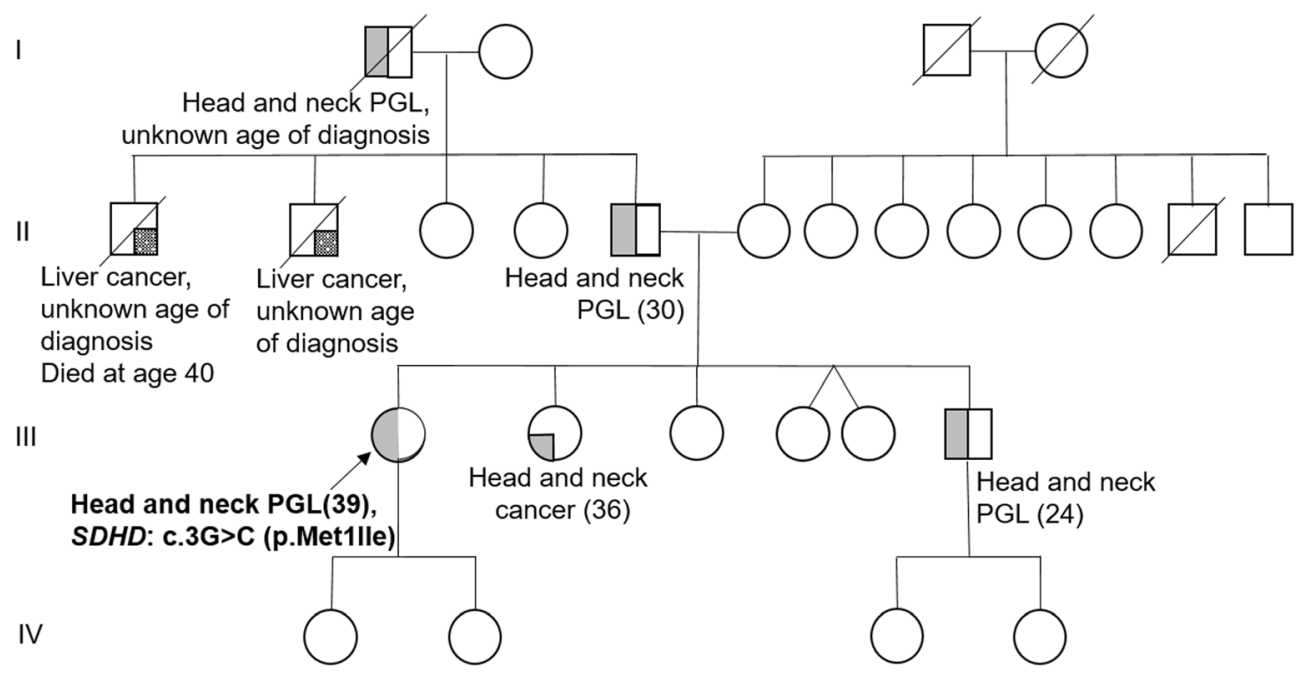

Fig. 1 Family pedigree of 3 patients with positive SDHD variants 


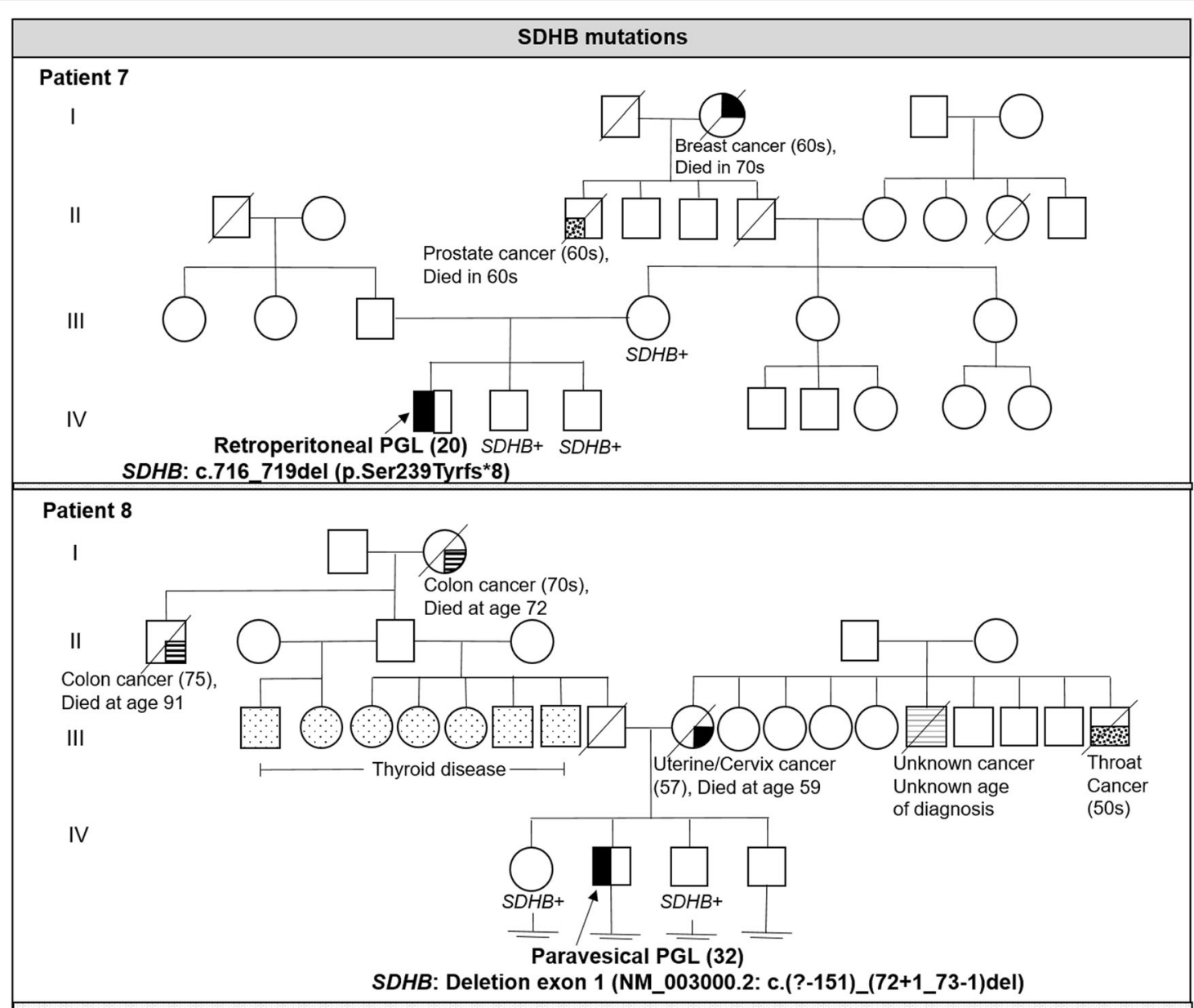

Patient 9

I

II
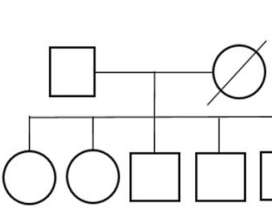

III
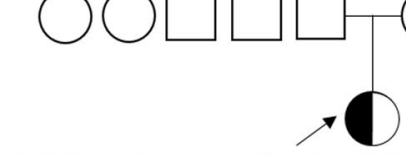

Renal PGL (13) and para-aortic PGL (25)

SDHB: c.756_765+4del
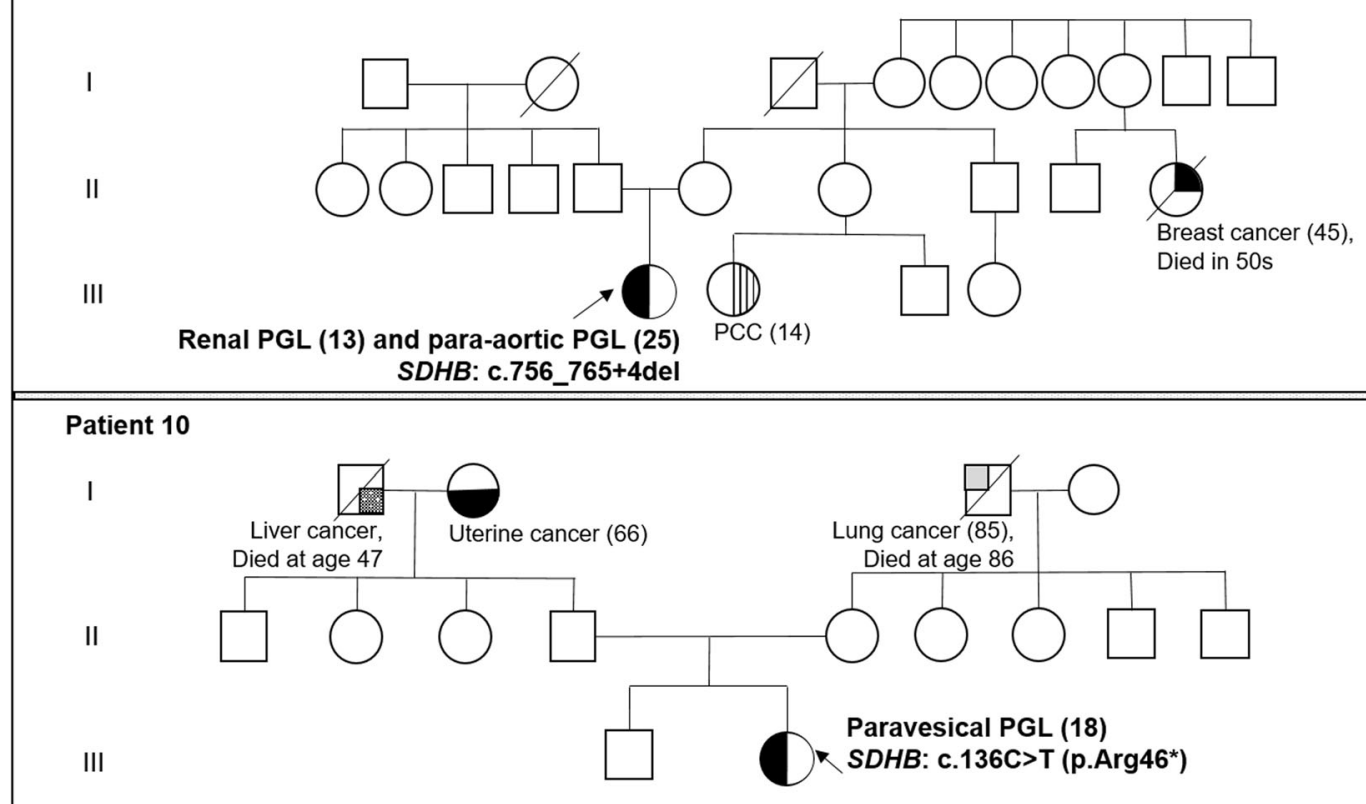

aravesical PGL (18)

SDHB: c.136C>T (p.Arg46*)

Fig. 2 Family pedigree of 4 patients with positive SDHB variants 
abdominal paraganglioma with majority involving the urogenital tract $(n=4)$ and one involving the retroperitoneal region. The remaining 2 patients (15.4\%) presented with adrenal pheochromocytoma alone. Median age at first presentation is 30 years (range 13-73). Ten patients (76.9\%) had young onset PGL/PCC at or before age 40, 4/13 patients (30.8\%) presented with multiple PGL/PCC tumours, and $3 / 13$ patients (23.1\%) had metastatic disease involving the bone, lung and liver. Two patients $(18.2 \%)$ had family history of paraganglioma $(n=1)$ or pheochromocytoma $(n=1)$.

Patients 1-3 presented with multifocal HN-PGLs with patient 1 also presenting with adrenal pheochromocytoma and were suspected clinically to have PGL1. Three patients (Patients 4-6) presented with non-multifocal HN-PGL with no other manifestations and were suspected clinically to have PGL1 or PGL3. Five patients (Patients 7-11) presented with extra-adrenal thoracoabdominal PGLs with two presenting with malignant disease. All five were clinically suspected to have PGL4. A differential diagnosis of Lynch syndrome was considered in Patient 11 in view of her personal history of multiple other malignancies including colorectal cancer. Patients 12 and 13 presented with adrenal pheochromocytoma with clinical features of hypersecretory catecholamines, with patient 13 presenting also with clear cell renal cell carcinoma, suspicious of PGL1, PGL4, or von Hippel-Lindau syndrome.

Ten of 13 patients (76.9\%) underwent genetic testing. Seven patients $(70.0 \%)$ were found to carry pathogenic variants $(S D H B=4, S D H D=3)$. No other pathogenic variants were identified. Patients who tested positive for $S D H B$ or $S D H D$ pathogenic variants were younger than those who tested negative (mean age $26 \pm 4$ vs $55 \pm 13$ years, $p=0.015$ ). All seven patients found with pathogenic variants were diagnosed with PGL/PCC clinically before age 40 . Three out of four patients who had multifocal tumour ( $n=3 / 4,75.0 \%)$, two of three patients who presented with metastatic disease $(n=2 / 3,66.7 \%)$, and both patients with family history of PGL/PCC $(n=2 / 2$, $100.0 \%)$ and who underwent genetic testing, tested positive. Three of 13 patients (23.1\%) did not undergo genetic testing as they were not keen to know the genetic information.

Among the seven patients found with pathogenic variants, 3 patients were found to carry pathogenic SDHD variants, including 2 Chinese patients and 1 Chinese-Myanmese patient. These three unrelated individuals all presented with head and neck paragangliomas, and were found to carry the same SDHD: c.3G > C (p.Met1Ile) variant that was previously reported to be a possible founder variant in Chinese [16-19]. Four patients were found to carry pathogenic $S D H B$ variants, and all four presented with extra- adrenal thoraco-abdominal paragangliomas, including 3 of the 4 patients with urogenital tract involvement. These 4 unrelated patients had different $S D H B$ variants, including three clearly pathogenic variants (large deletion $=1$, nonsense variant $=1$, frameshift variant leading to premature stop signal $=1$ ) and a possibly pathogenic splice donor variant. All $S D H B$ variants identified exist in ClinVar.

Two adult family members of Patient 7 and three adult family members of Patient 8 who were diagnosed with pathogenic $S D H B$ variants underwent cascade testing; 3 were confirmed to carry the familial $S D H B$ pathogenic variants (mean age 35 years, range 28-42). All 3 family members were asymptomatic and cancer free, including, of note, the 42-year old mother of Patient 7.

\section{Discussion}

Hereditary paraganglioma or pheochromocytoma syndromes are rare conditions, with fewer than $1 \%$ of patients who were referred to our adult cancer genetics clinic fulfilling clinical suspicion for hereditary PGL/ PCC. While $S D H B, S D H C$ and $S D H D$ genes are the classical causative genes of hereditary PGL/PCC, advancements in genetic analysis have revealed rarer causative genes such as SDHA, SDHAF2, MAX and TMEM127. The Task Force committee consisting of members from The Endocrine Society, European Society of Endocrinology, and American Association for Clinical Chemistry have reviewed the diagnostic algorithms from various studies and has recommended an optimal strategy for genetic screening, generally prioritizing younger age individuals, those with positive family history, and/or multifocal PGL/PCC, to undergo genetic tests [20]. Individuals with clinically high-risk features who do not show variant in the classic genes should be tested for the rarer genes [15], while targeted germline variant testing of RET, neurofibromatosis type 1 (NF1) or von HippelLindau syndrome $(V H L)$ are considered for individuals with syndromic presentation.

Although hereditary PGL/PCC is a fairly distinct entity, several of our patients report family history of other carcinomas that are unrelated to $\mathrm{PGL} / \mathrm{PCC}$, including breast, colorectal, prostate, and leukaemia, suggesting differential diagnoses such as $B R C A 1 / 2$ hereditary breast cancer syndrome, hereditary colorectal cancer syndrome, and Li Fraumeni syndrome. This highlights the benefits of next-generation sequencing multigene panel testing in these patients that encompasses not only genes associated with hereditary PGL/PCC syndrome but also causative genes of other adult hereditary cancer syndromes. In our highly selected population, $70 \%$ of patients tested were found to carry pathogenic variants confirming the diagnosis of hereditary PGL/PCC. Interestingly, despite the broad-based testing approach, pathogenic variants 
were only identified in the two most important $S D H$ genes, namely $S D H D$ and $S D H B$, with no pathogenic variants identified in any other PGL/PCC causative genes or other hereditary cancer genes. This observation is largely attributed to the small sample size, but also ascertains the importance of the $S D H D$ and $S D H B$ genes as causative genes of hereditary PGL/PCC in Asia. This has been similarly reported in another study conducted in Singapore, in which 5 of 7 patients with suspected hereditary PGL/PCC who tested positive carried SDHD $(n=2)$ or $S D H B(n=3)$ pathogenic variants, with the remaining two patients harbouring $V H L$ pathogenic variants [21].

Among the seven patients who were found with pathogenic germline variants in our series, 3 unrelated patients carried the same SDHD: c.3G >C (p.Met1lle) variant, while 4 patients were found with different $S D H B$ variants. $S D H D$ and $S D H B$ variant carriers have distinct characteristics, with $S D H D$ carriers typically presenting with head and neck PGLs that are rarely malignant, and $S D H B$ carriers having lesions involving mostly extraadrenal non-head and neck sites that behave more aggressively. Consistent with many other reports [21-23], patients who have younger onset presentation, multiple tumours, metastatic disease or family history of PGL/ PCCs, were more likely to be diagnosed with pathogenic $S D H$ variants in our study. Interestingly, four-fifths of patients in our series found with pathogenic $S D H B$ variant presented with urogenital PGL (renal = 1, bladder = $3)$; three were Chinese and the other was of mixed Chinese-Myanmese heritage. While genitourinary paragangliomas have been described, it is uncommon and comprises only $6.7 \%$ of all PGL cases in the US population [24]. Among urogenital PGLs, bladder (83.3\%) is the commonest site followed by other sites like renal, renal pelvis or spermatic cord. In the Surveillance, Epidemiology, and End Results (SEER) database, genitourinary PGLs are frequently seen in male and younger individuals, consistent with what we observed in our study [24]. A study conducted in London on patients with bladder paraganglioma found pathogenic $S D H B$ variants in 6/9 individuals, again mostly in male and younger individuals [25]. Although there have been several case series on urogenital paragangliomas in Asia, there is limited information on causative germline variants of these rare tumours [26, 27]. In the few Asian genetic studies on urogenital paragangliomas, $S D H B$ exon 7 deletion was reported in an Indian patient with bladder paraganglioma [28], while SDHB: c.112delC (p.Arg38fs) variant was reported in a Hong Kong Chinese patient with recurrent metastatic bladder paraganglioma [29]. Contrary to our study in which three of four patients who tested positive for pathogenic $S D H B$ variants had urogenital PGL, a Korean study of 2 patients with pathogenic $S D H B$ variants did not report any urogenital paraganglioma involvement [30]; instead, both patients presented with adrenal pheochromocytoma with one of them behaving in a malignant manner. More studies are required in Asia to determine if urogenital paragangliomas due to $S D H B$ variants are more common in certain ethnic populations.

In our series, only $15.4 \%(2 / 13)$ patients reported family history of PCC and/or PGL. Yet, 70\% (7/10) patients who underwent genetic testing were confirmed to carry pathogenic $S D H D$ or $S D H B$ variants. Both patients with family history of PGL/PCC tested positive, underscoring the importance of family history as a predictor for pathogenic SDH variants. The low proportion of reported family history may be due to small family sizes, ascertainment bias, and maternal genomic imprinting reported with some $S D H$ genes, and highlights the importance of not relying only on family history to select individuals for hereditary PGL/PCC testing in the clinic.

Separately, our series had identified several asymptomatic first-degree relatives to be $S D H B$ variant carriers from two families (Patients 7 and 8). Of note, Patient 7 was a 20-year old Filipino male who presented with retroperitoneal paraganglioma associated with hypertension from raised noradrenaline and normetanephrines but reports no family history of PGL or PCC. Upon confirmation of a pathogenic $S D H B$ variant in him, his cancer-free mother, aged 42, underwent cascade testing and was confirmed to carry the same variant, providing evidence of the maternal origin of the variant. Patient 8 is a 32-year old Chinese male who presented with paravesical paraganglioma associated with hypertension from raised nonadrenaline and normetapherines and again reported no definitive family history of PGL/PCC. Although his parents were not directly tested, two of his adult siblings subsequently tested positive for the same variant, confirming that the variant must have been inherited rather than occurring de novo. Recent studies have reported a lower range of penetrance of $25-50 \%$ in $S D H B$ variant carriers after taking into account the ascertainment of variant carriers [31,32]. The lower penetrance may be another reason for the lack of family history in the majority of patients seen in our series.

$S D H$ variants are generally distributed along the entire genes, with no obvious hot spots. However, a small number of founder variants in the $S D H$ genes that occur in high frequencies in certain geographically or culturally isolated groups of people have been reported, mostly amongst the European population. For example, $S D H D$ founder variants were reported amongst the Dutch (SDHD: c.274G > T (p.Asp92Tyr)) [33, 34], Polish (SDHD: c.33C > A (p.Cys11*)) [35], and Italian populations (SDHD: c.325C > T (p.Gln109*)) [36], while an $S D H B$ founder variant (SDHB: c.201-4429_287-933del 
(p.Cys68Hisfs"21)) has been reported in the Dutch population [37]. These founder variants have generated considerable interest, because they might direct testing strategy towards specific prevalent founder variants in certain population. In comparison, there have been limited reports of SDH founder variants in Asians. Zha et al reported the $S D H D$ : c.3G $>\mathrm{C}$ (p.Met1Ile) variant as a possible founder variant in the Chinese population [17], with haplotype analysis showing three out of four unrelated Chinese families carrying the SDHD: c.3G $>\mathrm{C}$ (p.Met1Ile) variant residing in China, Singapore [16] and Hong Kong [18], to share a common haplotype spanning a $280 \mathrm{~kb}$ region. In our current study, all three patients who were diagnosed with $S D H D$ pathogenic variants carried the same SDHD: c.3G >C (p.Met1Ile) variant, which results in abolishment of the initiation codon. These three unrelated patients were of Chinese descent, although they live in different parts of South-East Asia: Singapore, Indonesia and Myammar, further supporting the previous observation that this variant is a common founder variant in Chinese. Interestingly, we were not initially aware of the Chinese ancestry of the Myanmese patient whose variant is likely paternal in origin as both her paternal grandfather and father presented with head and neck paragangliomas. After she was diagnosed to carry the $S D H D$ : c.3G $>$ C (p.Met1lle) variant, further questioning revealed that she was of mixed ChineseMyanmese ancestry, with her affected paternal grandfather being Chinese. Maternal imprinting has been reported for $S D H D$ variants, and the clinical manifestation of PGL in this index patient whose variant is paternal in origin is consistent with this notion. The patient as well as her affected brother each has two teenage children who can eventually undergo predictive testing; it will be of interest to follow up this family closely to determine the transmission pattern of the SDHD: c.3G >C (p.Met1Ile) variant.

\section{Conclusion}

Hereditary PGL/PCC is rare, accounting for around $0.6 \%$ of patients encountered in an adult cancer genetics clinic in Asia. Fewer than 20\% of patients report family history of PGL/PCCs, highlighting the importance of other clinical features such as young age at diagnosis, multiple tumours and metastatic disease to identify high-risk individuals for genetic testing. The increasing availability of multi-gene panel testing with next generation sequencing has facilitated the diagnosis of pathogenic variants in these individuals. $S D H D$ and $S D H B$ genes remain the most important causative genes of hereditary PGL/PCC in Asia, with an SDHD founder variant existing in Chinese head and neck PGL families who reside in different countries in Asia.

\section{Acknowledgements}

Not applicable.

\begin{abstract}
Authors' contributions
Soo Chin Lee has conceived and designed the study. Soo Chin Lee and Kay Reen Ting contributed to the writing of the manuscript. Soo Chin Lee, Kay Reen Ting, Pei Yi Ong and Samuel Ow Guan Wei contributed to the analysis and interpretation of the results. Soo Chin Lee, Samuel Ow Guan Wei and Pei Yi Ong were responsible for data collection. Rajeev Parameswaran, Chin Meng Khoo and Doddabele Srinivasa Deepak provided critical feedback of the manuscript. All authors provided critical feedback and helped shape the research, analysis and manuscript. All authors have approved the final article.
\end{abstract}

Funding

Not applicable.

\section{Availability of data and materials}

All data generated or analyzed during this study are included in this published article and its supplementary information files.

Ethics approval and consent to participate

Not applicable. No tests or experiments were performed on humans as part of this study.

Consent for publication

Not applicable.

\section{Competing interests}

The authors declare that they have no other competing interests.

\section{Author details}

${ }^{1}$ Department of Haematology-Oncology, National University Cancer Institute, Singapore (NCIS), National University Health System, 1E Kent Ridge Road, Singapore 119228 , Singapore. ${ }^{2}$ Yong Loo Lin School of Medicine, National University of Singapore, Singapore, Singapore. ${ }^{3}$ Department of Surgery, Division of General Surgery (Thyroid and Endocrine Surgery), National University Hospital, Singapore, Singapore. ${ }^{4}$ Department of Medicine, Division of Endocrinology, National University Hospital, Singapore, Singapore. ${ }^{5} \mathrm{Cancer}$ Science Institute, Singapore, Singapore.

Received: 18 August 2020 Accepted: 26 November 2020

Published online: 11 December 2020

\section{References}

1. Beard CM, Sheps SG, Kurland LT, et al. Occurrence of pheochromocytoma in Rochester, Minnesota, 1950 through 1979. Mayo Clin Proc. 1983:58:802-4.

2. Berends AMA, Buitenwerf $E$, de Krijger RR, et al. Incidence of pheochromocytoma and sympathetic paraganglioma in the Netherlands: a nationwide study and systematic review. Eur J Intern Med. 2018;51:68-73.

3. Lam AK-Y. Update on adrenal Tumours in 2017 World Health Organization (WHO) of endocrine Tumours. Endocr Pathol. 2017;28:213-27.

4. Chrisoulidou A, Kaltsas G, Ilias I, et al. The diagnosis and management of malignant pheochromocytoma and paraganglioma. Endocr Relat Cancer. 2007;14:569-85.

5. Tischler AS, Pacak K, Eisenhofer G. The adrenal medulla and extra-adrenal Paraganglia: then and now. Endocr Pathol. 2014;25:49-58.

6. Lee J, Barich F, Karnell L, et al. National Cancer Data Base Report on malignant Paragangliomas of the head and neck. Cancer. 2002;94:730.

7. Goffredo P, Sosa JA, Roman SA. Malignant pheochromocytoma and paraganglioma: a population level analysis of long-term survival over two decades. J Surg Oncol. 2013;107:659-64.

8. Hamidi O, Young WF, Gruber L, et al. Outcomes of patients with metastatic phaeochromocytoma and paraganglioma: a systematic review and metaanalysis. Clin Endocrinol. 2017:87:440-50.

9. Favier J, Amar L, Gimenez-Roqueplo A-P. Paraganglioma and phaeochromocytoma: from genetics to personalized medicine. Nat Rev Endocrinol. 2015;11:101-11.

10. Buffet A, Venisse A, Nau V, et al. A decade (2001-2010) of genetic testing for pheochromocytoma and paraganglioma. Horm Metab Res. 2012;44:359-66. 
11. Else T, Greenberg S, Fishbein L. Hereditary ParagangliomaPheochromocytoma syndromes. Gene Rev. University of Washington, Seattle; 2018.

12. Neumann HPH, Bausch B, McWhinney SR, et al. Germ-line mutations in Nonsyndromic Pheochromocytoma. N Engl J Med. 2002;346:1459-66.

13. Neumann HPH, Young WF, Eng C. Pheochromocytoma and Paraganglioma. Longo DL, editor. N Engl J Med. 2019;381:552-65.

14. Benn DE, Robinson BG, Clifton-Bligh RJ. 15 years of paraganglioma: clinical manifestations of paraganglioma syndromes types 1-5. Endocr Relat Cancer. 2015;22:T91-103.

15. Bausch B, Schiavi F, Ni Y, et al. Clinical characterization of the Pheochromocytoma and Paraganglioma susceptibility genes SDHA, TMEM127, MAX, and SDHAF2 for gene-informed prevention. JAMA Oncol. 2017;3:1204-12.

16. Lee S-C, Chionh S-B, Chong S-M, et al. Hereditary Paraganglioma due to the SDHD M1I mutation in a second Chinese family: a founder effect? Laryngoscope. 2003;113:1055-8.

17. Zha $Y$, Chen $X$, Lam $C$, et al. Is the $C .3 G>C$ mutation mutation in the succinate dehydrogenase subunit $D(S D H D)$ gene due to a founder effect in Chinese head and neck paraganglioma patients? Laryngoscope. 2011;121: 1760-4.

18. Ma R, Lam C, Chan W, et al. A Chinese family with familial paraganglioma syndrome due to succinate dehydrogenase deficiency. Hong Kong Med J. 2007;13:151-4

19. Zhu WD, Wang ZY, Chai YC, et al. Germline mutations and genotype-phenotype associations in head and neck paraganglioma patients with negative family history in China. Eur J Med Genet. 2015:58:433-8.

20. Lenders JWM, Duh Q-Y, Eisenhofer G, et al. Pheochromocytoma and Paraganglioma: an Endocrine Society clinical practice guideline. J Clin Endocrinol Metab. 2014;99:1915-42.

21. Hong Wern Chew W, Courtney E, Hing Lim K, et al. Clinical management of pheochromocytoma and paraganglioma in Singapore: missed opportunities for genetic testing. Mol Genet Genomic Med. 2017;5:602-7.

22. Burnichon $N$, Rohmer $V$, Amar $L$, et al. The succinate dehydrogenase genetic testing in a large prospective series of patients with paragangliomas. J Clin Endocrinol Metab. 2009;94:2817-27.

23. Mannelli M, Castellano M, Schiavi F, et al. Clinically guided genetic screening in a large cohort of Italian patients with Pheochromocytomas and/or functional or nonfunctional Paragangliomas. J Clin Endocrinol Metab. 2009;94:1541-7.

24. Purnell S, Sidana A, Maruf M, et al. Genitourinary paraganglioma: Demographic, pathologic, and clinical characteristics in the surveillance, epidemiology, and end results database (2000-2012). Urol Oncol. 2017;35: 457.e9-457.e14

25. Srirangalingam $U$, Banerjee $A$, Patki $P$, et al. Succinate dehydrogenase $B$ (SDHB)-associated bladder Paragangliomas. Clin Genitourin Cancer. 2017;15: e131-6.

26. Maeda M, Funahashi $Y$, Katoh $M$, et al. Malignant bladder pheochromocytoma with SDHB genetic mutation. Aktuelle Urol. 2013;44: 381-2.

27. Park S, Kang SY, Kwon GY, et al. Original articles Clinicopathologic characteristics and mutational status of succinate dehydrogenase genes in Paraganglioma of the urinary bladder a multi-institutional Korean study. Arch Pathol Lab Med. 2017;141:671-7.

28. Pai R, Ebenazer A, Paul M, et al. Mutations seen among patients with Pheochromocytoma and Paraganglioma at a referral center from India. Horm Metab Res. 2015;47:133-7.

29. Lee C, Cheung C, Chow W, et al. Genetics of apparently sporadic Pheochromocytoma and Paraganglioma in a Chinese population. Horm Metab Res. 2015;47:833-8.

30. Kim JH, Seong M-W, Lee $\mathrm{KE}$, et al. Germline mutations and genotypephenotype correlations in patients with apparently sporadic pheochromocytoma/paraganglioma in Korea. Clin Genet. 2014;86:482-6.

31. Jafri M, Maher ER. The genetics of phaeochromocytoma: using clinical features to guide genetic testing. Eur J Endocrinol. 2012;166:151-8.

32. Schiavi F, Milne RL, Anda E, et al. Are we overestimating the penetrance of mutations in SDHB? Hum Mutat. 2010;31:761-2.

33. Taschner PEM, Jansen JC, Baysal BE, et al. Nearly all hereditary paragangliomas in the Netherlands are caused by two founder mutations in the SDHD gene. Genes Chromosom Cancer. 2001;31:274-81.
34. Hensen EF, van Duinen N, Jansen JC, et al. High prevalence of founder mutations of the succinate dehydrogenase genes in the Netherlands. Clin Genet. 2012:81:284-8.

35. Peczkowska M, Erlic Z, Hoffmann MM, et al. Impact of screening kindreds for SDHD p.Cys $11 \mathrm{X}$ as a common mutation associated with paraganglioma syndrome type 1. J Clin Endocrinol Metab. 2008;93:4818-25.

36. Simi L, Sestini R, Ferruzzi $P$, et al. Phenotype variability of neural crest derived tumours in six Italian families segregating the same founder SDHD mutation Q109X. J Med Genet. 2005;42:e52.

37. Bayley J-P, Grimbergen AEM, van Bunderen PA, et al. The first Dutch SDHB founder deletion in paraganglioma-pheochromocytoma patients. BMC Med Genet. 2009;10:34

\section{Publisher's Note}

Springer Nature remains neutral with regard to jurisdictional claims in published maps and institutional affiliations.

\section{Ready to submit your research? Choose BMC and benefit from:}

- fast, convenient online submission

- thorough peer review by experienced researchers in your field

- rapid publication on acceptance

- support for research data, including large and complex data types

- gold Open Access which fosters wider collaboration and increased citations

- maximum visibility for your research: over $100 \mathrm{M}$ website views per year

At $\mathrm{BMC}$, research is always in progress.

Learn more biomedcentral.com/submissions 